\title{
Reinventing the Future: A Study of the Organizational Mind
}

\author{
Isabel Ramos, João Álvaro Carvalho \\ Information Systems Department, University of Minho, Campus de \\ Azurém, 4800-058 Guimarães, Portugal \{iramos, jac\}@dsi.uminho.pt
}

\begin{abstract}
This paper describes the concept of the self- and metarepresentation capabilities of the organization, a constituent of what we call the organizational mind. Our claim is that these capabilities are responsible for the emergence of a collective self that is of central importance in the formation of the organizational identity. These capabilities are relevant to the information systems field, as Information Technology applications play a central role in the support of those representational capabilities. The paper presents a summary of a theoretical perspective that supports a research project aimed at developing a framework to guide managers diagnosing identity dysfunctions resulting from impaired representational capabilities of the organization.
\end{abstract}

\section{Introduction}

This paper focuses on the self- and meta-representation capabilities of organizations, a constituent of what we call the organizational mind. Our claim is that these capabilities are responsible for the emergence of a collective self that is of central importance in the formation of organizational identity. These capabilities are relevant to the information systems field as Information Technology (IT) applications play a central role on the support of those representational capabilities.

The idea that an organization forms and uses representations of itself and of relevant external entities with which it interacts as well as meta-representations derived from self-representations, is based on an analogous phenomenon occurring in the human mind. Human representational capabilities are herein taken as a metaphor to explain the organization's ability to think and act as a coherent whole a collective and distributed self. 
Although the exploration of this metaphor might demand empirical work, we believe that it constitutes a plausible and promising foundation to be applied in organizations for diagnosing the origins of performance problems arising from identity dysfunctions and for defining structural requirements for successful performance.

Section 2 provides the context for the representational capabilities of the organization - the organizational mind framework. It is not the purpose of this paper to present the details of current knowledge about the human mind developed in Neurosciences due to the limited space of a paper. Thus, in section 2.1 , we provide only an overview of the main concepts supporting the metaphor from which we will develop the organizational mind framework. Section 2.2 , we identify specific capabilities of the human mind that will be used to locate and study equivalent capabilities of the organization. These organizational capabilities will be approached as complex collective constructs that will be incrementally developed and validated in multilevel research and theory development [1].

Section 3 details the concept of self- and meta- representation capabilities and states its relevance for organizations, the first multilevel construct to be studied. In Section 3, we briefly describe how current and emerging IT may be used to leverage the representational capabilities of the organization.

\section{Representational Capabilities and the Organizational Mind}

The representational capabilities of organizations are only one aspect of a comprehensive framework we designate as the organizational mind that we aim to develop further and whose usefulness we expect to demonstrate in a broader and interdisciplinary research program. The organizational mind concept will be developed and operationalized by defining the organizational equivalent to human perception, cognition, emotion, and consciousness. This concept will relate organizational aspects such as structure, human resources management, power, culture, strategy, change management, leadership, innovation, learning, and IT applications. The interconnection of all these aspects may lead to an integrated view of distributed perception, cognition, emotion, and consciousness in organizations.

Our approach does not imply reifying the organizational mind but to use those human-mind-centered concepts metaphorically to establish the basis for an explanation of how the organization's members collectively gather information from the environment and from its interior, interpret it, build an image of the organization as a whole, recognize the organization's problems and needs, project its action and construct its future.

\subsection{Human Mind}

According to scientific knowledge produced in the neurosciences (e.g., [2-5]), the human mind emerges from the brain devices that support the mental processes of: 
Perception. This mental process enables human beings to know external objects and events, events happening inside the body, mental objects such as thoughts and modes of thinking, and of the self in relation to perceived physical or mental objects.

Cognition. This mental process includes aspects such as vigil, production of thoughts, attention, memory, language, and reason.

Emotion. This mental process is built from simple and automatic responses to competent stimuli. Emotions are specific repertoires of action that help an individual to achieve the circumstances conducive to survival and well-being.

Consciousness. This mental process enables the knowing of an object or action that can be ascribed to the self. The nuclear consciousness provides the individual with a sense of the self, the now, and the here. The extended consciousness provides a complex sense of self, corresponding to an individual's identity. Consciousness permits also the knowledge of an individual's history, and his or her past and anticipated future, and enables him or her to stay alert to the surrounding world.

In conjunction, these mental processes give rise to mental phenomena such as:

Feelings. Mental images that assist in making choices regarding self-preservation. They are the expression of emotions at the mind level. Feelings help us to solve nonstandard problems involving creativity, judgment, and decision-making, requiring the display and manipulation of vast amounts of knowledge. Feelings can help or impede learning and recall.

Learning. The construction of new knowledge about the world that surrounds an individual and her or his role in that world, and about the self and its own potential for action and interaction. It is intimately related with life experience. Learning changes the way the individual perceives, thinks, and behaves in order to accommodate new experiences.

Intelligence. The capacity to manipulate knowledge for the planning and execution of new answers to any problem perceived as endangering survival or well-being.

Creativity. The capacity to produce new ideas and new things. It is rooted in the cognitive functions of memory, reason, and language and is informed by the revelations of consciousness.

The above processes and phenomena support human action and its adaptive characteristics They demand the existence of representations that enable humans to exercise self-control in social situations and to use the "I" in a fluent and correct way, know the current body configuration and status, engage in self-imagery, identify feelings such as happiness, and show sympathy with the distress of others [2].

Such representations include (i) the internal milieu and viscera via chemical and neural pathways, (ii) musculoskeletal structures, (iii) autobiographical events, (iv) causal and simulation models of the relevant aspects of a body's movable parts, the relations between them, the relations to its sensory input, and its goals, (v) causal and simulation models of the social world in which it finds itself, and (vi) where one is in space-time and the social order.

Disturbances in the self- and meta- representation capabilities of humans may result in inability to acquire new knowledge, loss of autobiographical information; inability to recognize thoughts as one's own thoughts, inability to recognize body parts as one's own, inability to inhibit unwise impulses, personality changes, reckless in decision-making, and social insensitivity. 
Representational damages impair identity [6]. Identity enables the integration of body-state signals, the evaluation of options, and the choice-making. Thus, humans are able to act as a coherent whole, not as a group of independent sub-systems with competing interests. Identity also permits the self/non-self distinction, and enables to distinguish between inner-world representations and outer-world representations and to build a meta-representational model of the relation between the outer and the inner

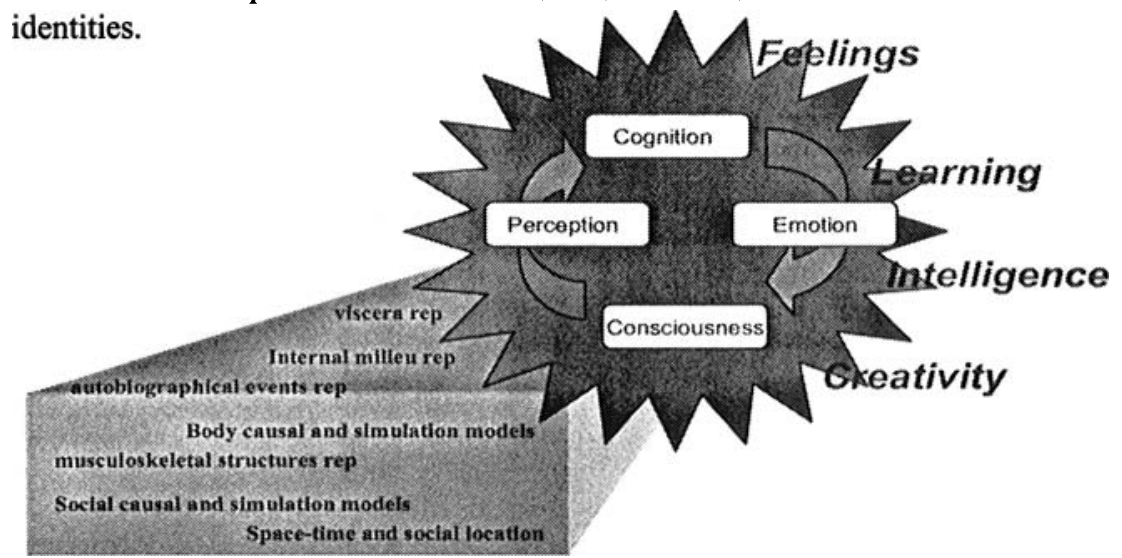

Fig. 1: Mental processes and supporting self- and meta- representations

\subsection{Organizational Mind: An Evolving Concept}

Terms such as knowledge management, organizational memory and identity, business intelligence, and organizational learning are becoming usual in the discourse of the social and organizational sciences. Each presupposes a parallel between the human mind and the organizational capability of intelligent manipulation of knowledge to support the planning and execution of new and better solutions to problems concerning survival and well-being. However, when the literature in the fields conveyed by those terms is analyzed, this parallel is lost in favor of sociological, economic, and technological views, often explored separately.

One of the earliest references to the term "organizational mind" is provided by Ian Mitroff, in his book "Stakeholders of the Organizational Mind" [7]. The organizational mind is equated with the collective thinking of organizational managers and the consequential management and organizing practices. In his book, Mitroff relates the organizational types with the personality types and ego states of top managers.

Other authors have brought the concept of organizational mind into relation with a shared understanding of strategic problems, competitive conditions, and the internal and external environments they face [8].

In general, this initial view presents the organizational mind as the global information processing system of the organization, which includes human and technological processors. This global system permits the access, transformation and delivery of information from a variety of perspectives. 
This view has evolved by integrating important insights from Psychology and Social Constructionism. Weick and Roberts [6] state that the heedful interactions between its members generate the collective mind of an organization. In order to be effective, newcomers must be socialized into the collective mind. The collective mind emerges from the joint production of thoughts (cognition) in the process of heedful action and interaction. This view brought to consideration organizational mindfulness, considered important to organizational learning $([9,10])$. Organizational mindfulness is local and situated, involves thinking in "real time" and is simultaneous with the execution of action. Thus, it involves both action and cognition. Organizational mindfulness enables the organization, as a whole, to reveal new opportunities in the ongoing activity and keep its action close to the defined plans and expectations.

Another view of the organizational mind emerged from the claims of complex systems theory [11]. The organization is seen as a self-organizing, adaptive, nonlinear, and complex system showing the following properties [12]:

Connectivity: resources, human and non-human, are interconnected; managers should think global and act locally.

Indeterminacy: although reality abides the law of causation, knowledge of the effect of any particular cause is an approximation and never a fact that can be known in advance; the how of change must be constructed as the change unfolds.

Dissipation: reality is the product and the framework of thinking, action and interaction. It is in permanent motion.

Emergence: Thinking, action, and interaction are continuously changing and producing emergent phenomena; managers should let go of command and control.

Consciousness: it is an emergent phenomenon in organizations and comprises the collective consciousness of every individual within the bounds of the organization.

The above properties support the emergence of organizational mind. The organizational mind is ever in motion. It is an emergent, distributed and transactional phenomenon conceiving shared thoughts and feelings, shaping desires, assembling plans, evaluating experience, interpreting perceptions, and initiating actions. Leadership is a discretionary role open for every employee rather than a fixed privilege of a particular hierarchical position in the organization.

The chaordic view of the organizational mind is often criticized for its lack of empirical research and by not providing an useful operationalization of the theoretical claims. Another critic is that there is a tendency to reify the organizational mind, seeking the social mechanisms responsible for the emergence of a new being or a meta-consciousness independent from the individual consciousnesses that give rise to it.

The above theoretical views of the organizational mind advance some important explanations for collective and distributed cognition and action in organizations, but they have not been successful in providing methodological tools to study the working of organizational mind or to improve its intelligence, learning ability or creativity. We consider that recent knowledge on human mind developed in Neurosciences may provide those methodological tools.

The next paragraphs of this section offer a first glance at the concepts that can be used to develop the organizational mind concept. This concept can then be used to develop the necessary tools to diagnose possible dysfunctions in the organizational 
mind and to improve its intelligence. Some of the ideas here expressed are already being explored separately by some authors ([13-15]).

The organizational mind comprises processes and phenomena corresponding to those found in the human mind. It is our expectation that the organizational mind constitutes an interesting and useful framework to assist the study of how collective experience is understood and memorized, highlighting the events and objects that organizations choose to pay attention to. It has also the potential to assist in understanding the language elements upon which the organizational experience is constructed and what processes enable organizations to reason about that experience. By constructing representations about itself and its environment, an organization, as a collective self, creates a sense of meaning about the world and its action in that same world. The organizational mind framework can be used to improve an organization's capabilities for survival and also to guide its members' search for their collective well-being.

Using the organizational mind framework, researchers and managers will be able to evaluate an organization's mental capabilities of:

- Intelligence - how its members use the available knowledge to plan and implement solutions to problems and environmental challenges, ensuring the organization's survival and well-being;

- Learning ability - how their members collectively accommodate new experience by changing the way they perceive, think and behave;

- Creativity - the organizations' capability to produce new ideas and new things to ensure a dynamic adaptation to the internal and environmental challenges and opportunities.

To consider the organizational mind concept presupposes to view an organization as a whole capable of coherent behavior and of holding some sense of a collective self.

\subsection{Representational Capabilities of Organizations: A Collective Construct}

Research constructs can be thought of as "conceptual notions whose existence must be inferred from more observable actions or features of an entity" [1]. Constructs that describe phenomena observed at the level of any interdependent and goaldirected combination of individuals, groups, organizational units, organizations or industries are collective constructs.

Collective constructs may be measured at various levels of analysis. The most elementary unit of analysis of any collective construct is the individual behavioural act. Individual action is influenced by a multitude of situational and contextual factors. Within a social system, the action of individuals meets each other in space and time, resulting in interpersonal interaction. "As interaction occurs within larger groups of individuals, a structure of collective action emerges that transcends the individuals who constitute the collective" [1]. In organizations, human interaction is mediated or made possible by IT applications. Therefore, IT applications are a key element of the structure of collective action.

A collective construct may manifest itself at several levels of analysis, presenting similarity of function but not of structure. The function of a construct refers to its 
causal outputs or effects. Functional analysis is the assessment of a construct's outputs in the organizational system.

The self- and meta- representation capability of organizations is a collective construct describing a phenomenon that can be observed at several levels of analysis. Individuals create representations of themselves as members of the organization they work for, their work interactions, their job history, [mental and material] models of the organization's social systems in which they are integrated, and their place in space-time and social order.

The actions of individuals are constrained and guided by the above representations. In the process of acting, individuals also create and transform representations. At the group level, collective action emerges that transcends the individuals who constitute the collective. Collective action that becomes recurrent, materially bounded and situated may be called practice [16]. This practice is mediated or enacted by IT applications.

As groups try to make sense of their actions within the context of the organization, they create and transform shared representations of themselves as social systems working within a larger social system, the organization. These are collective self- and meta-representations mediating the perceptions of group members and the understanding of their reality [16].

The organization is the highest level of analysis considered in our research. The structure of the construct is similar to the structure of the construct at the level of organizational groups. The only difference is that we have to consider groups and inter-groups practices and representations as well as representations of relevant entities outside the organization and of organizational interactions with those entities.

Self- and meta-representation capabilities at all levels in the organization enable the acquisition of new knowledge, production and retention of autobiographical information, recognition of thoughts as one's own thoughts, define boundaries and recognize the constituent of one's social body, inhibit unwise impulses, create a sense of personality continuity, produce coherent decision making and create social sensitivity. The representational capabilities are the main holders of identity.

One last point that must be considered in our research is that, at all levels of analysis, the process of self- representing is shaped by contextual dimensions such as structural, political and symbolic characteristics of the larger social system within which the object of analysis operates.

The organizational self- and meta- representation capabilities imply that the organization is capable of forming and using representations of itself and of the interactions with relevant external entities (self-representations), and representations built upon self-representations (meta-representations). Self- and metarepresentations address (i) what is "perceived", moment-by-moment, as happening in its internal and external environments; (ii) its envisaged future; (iii) past experience; (iv) its structure, causal, and process models of its internal workings; (v) structure, causal and process models of its social and economic environment; (vi) presumed image held by relevant stakeholders about the organization.

Accordingly, disturbances in the representational capabilities of an organization may result in inability to acquire new knowledge, loss of autobiographical information, inability to define shared insights, concepts, and motivations, inability 
to recognize organizational units as integrating parts of a whole, inability to agree to sanctioned action, inability to set up consistent patterns of decision and action, and environmental and social insensitivity.

Organizational identity may also be impaired by representational damages $([6,17])$. Although there are obvious and fundamental differences between how identity and representations are implemented in humans and organizations [18], there are also important conceptual similarities that can be explored, contributing to a more precise conceptualization of the organizational identity [19].

\section{Why Monitoring the Representational Capabilities of the Organization is Important}

In humans, the representational capabilities are essential to intelligent behavior since they are the foundation of human identity. Likewise, we can assume these capabilities are essential to organizational intelligent behavior because they are the foundation of organizational identity.

An organization's representational capabilities are distributed by different organizational actors, both human and automatic. Furthermore, as these representations have multiple usages they are relevant to very different organizational activities and phenomena. Finally, the different nature of these representations imply different ways of creating, storing and retrieving them..

All these aspects of organizations' representational capabilities justify that they are cared for in organizations. This is actually already happening. However, the organizational activities/processes that deal with representational capabilities are independent from each other, therefore concealing the common aspects and object of these different activities.

\subsection{Organizational Identity}

Organizational identity has been the focus of much work in organizational sciences, psychology, and management sciences. Organizational identity is particularly relevant since our research addresses a key component of identity formation, the self- and meta- representations required for the emergence of a collective and distributed sense of self. While this is a reasonably unexplored organizational phenomenon, some efforts have already been developed ([18-22]).

- Self-categorization theory focus on the self-categorization of individuals as members of groups;

- Symbolic interactionism focuses the construction of the self from the representations individuals and groups hold about the perceptions of others.

- Studies of communities of practice, role playing theory, structuration theory, and communication theory focus the construction of self-representations through interaction inside and with the outside environment of the organization.

- Narrative and discourse analysis focus the elicitation of self-representations and their reformulation through organizational dialogues.

- Institutional theory focuses stable self-representations. 
- Boundary phenomenology addresses the concept of autopoietic unity as a metaphor to explore issues of organizational identity and integrity.

- Theories of shared mental models and transactive memories focus how selfrepresentations are held in organizations.

- Information Systems theories have been highlighting the requirements for systems and technological architectures that effectively support representations and meta-representations of the collective self.

\subsection{Information Technology Support to Organizational Representation Capabilities}

IT applications constitute one of the key components of the representational capabilities of an organization. This section provides a first approach to the IT's role in leveraging an organization's ability to create, preserve, and transform representations. Table 1 presents some types of IT applications and the representations that they deal with. For the purposes of this presentation, we regrouped self- and meta- representations in 5 types.

Moment-by-moment organizational experience: the transactions, operations, measurements, and communication happening in all levels of the organization which are mediated or enacted by the system. Envisaged future: goals, strategies, forecasts, and objectives stored or defined with the help of the system. Past experience: patterns of behavior, decision or interaction with stakeholders elicited by the system. Structural, process, and causal models: models of the organization itself, models of the organization's business and market, and models of the economic, social or political environments. These models include the relevant parts of each of those domains, the relations between the parts, the relations of the parts with the inputs of the domain, and the domain's goals. These models may be produced by information systems and used to simulate and predict behavior as well as to define the appropriate action to avoid or solve problems and to take advantage of opportunities. Organizational image: the image that organizational members believe relevant stakeholders, business partners or competitors have of their organization and that information systems help shaping.

Table 1. Representations created or handle by information systems used in organizations

\begin{tabular}{cccccc}
\hline & $\begin{array}{c}\text { Moment-by- } \\
\text { moment } \\
\text { experience }\end{array}$ & $\begin{array}{c}\text { Envisaged } \\
\text { future }\end{array}$ & $\begin{array}{c}\text { Past } \\
\text { experience }\end{array}$ & Models & $\begin{array}{c}\text { Perceived } \\
\text { organizational } \\
\text { image }\end{array}$ \\
\hline Just-in-time inventory systems & $\mathbf{X}$ & & & $\mathbf{X}$ & $\mathbf{X}$ \\
Value Chain Mng Systems & $\mathbf{X}$ & & $\mathbf{X}$ & $\mathbf{X}$ & $\mathbf{X}$ \\
$\quad$ E-business Systems & $\mathbf{X}$ & $\mathbf{X}$ & & $\mathbf{X}$ & \\
Decision Support Systems & & $\mathbf{X}$ & $\mathbf{X}$ & $\mathbf{X}$ & \\
Business Intelligence Systems & & & & & \\
Human Capital Systems & & $\mathbf{X}$ & $\mathbf{X}$ \\
Systems to Support & $\mathbf{X}$ & & $\mathbf{X}$ & & \\
Communication & $\mathbf{X}$ & $\mathbf{X}$ & & & \\
Collaborative Systems & & & &
\end{tabular}


Just in time inventory systems hold representations of the innards of an organization, the stored items and their quantities as well as the needs for new items or their arrival. At the heart of these systems is a certain model of supplier behavior and of the organization's interaction with suppliers.

Value chain management systems hold representations of the internal workings of the organization and its interactions with suppliers and customers. They can also elicit patterns of behavior and of interaction with suppliers and clients, and simulate their reactions to different organizational initiatives. Finally, these systems help organizational members, either intentionally or unintentionally, projecting an image of their organization.

E-business systems register transactions and communication. They also serve as gateways to the internal workings of the organization, thus helping to define its boundaries. They help form a view of the past experience of the organization by linking different kinds of stored representations. E-business systems also help shaping the organizational image.

Decision support systems, by allowing the simulation of decisions, help organizational members envisage possible futures for the organization or of an organizational unit. They are rooted in causal models of the organization or relevant domains of its environment. More sophisticated systems of this kind generate these models.

Business Intelligence Systems, with their typical data mining tools, support the elicitation of the past experience of an organization and generate causal models.

Systems that support communication register and deliver representations of the thinking and action, both of organizational members and external entities that communicate with them.

Collaborative systems support the moment-by-moment thinking and action of work groups, helping them to form views of the organizational past and decide for the future.

The categories of systems included in the table were chosen for their widespread use, facilitating the understanding of their role in the creation and transformation of organizational self- representations. In our future work, we intend to develop a systematic mapping of the information systems categories together with the representations they handle. The table also emphasizes the possibility of an organization having several, possibly conflicting, representations of the same object, either physical or conceptual.

Our research will present new ways of using present IT to leverage the representational capabilities of an organization, therefore improving the collective capabilities of problem solving, decision making, adaptation to changing conditions, and construction of a common future.

\section{Conclusion}

This paper focuses on one of the aspects of what we called the organizational mind: the self- and meta- representation capabilities of the organization. We described the concept as defined for the human mind and tried to build a bridge to what may be 
similar capabilities in organizations. In doing this we are assuming that such organizational capabilities are responsible for the emergence of a collective self which is of key importance for the ability of the organization to act coherently and intelligently in response to internal or external threats to its survival and well-being.

In future research, we intend to verify the propositions we are assuming in this paper, namely, that it is possible to define the requirements of healthy representational capabilities for the organization and that disturbance in these capabilities may lead to identity dysfunctions that negatively affect the organization's performance.

At the Information Systems Department of the University of Minho (Portugal), we are starting a research project within the lines of thought described in this paper. The objectives of the project are:

1. To define the concepts of "organizational self-representation capability" and "organizational meta-representation capability", using the human capability for self-representation and meta-representation as the supporting metaphor.

2. To define the structural, socio-cultural, and technological components of the above concepts in organizations.

3. To design an architecture for the organizational key components of the concept.

4. To develop key performance indicators to measure the maturity of the self- and meta- representation capabilities of the organization.

5. To define a model linking the organizational representational capabilities to the emergence of organizational identity.

6. To create a method and a prototype of a supporting computer based tool to assist (a) the diagnosis of potential identity dysfunctions related with problems in representational capabilities of the organization, and (b) the planning of effective interventions to reduce the diagnosed dysfunctions.

\section{References}

1. F. P. Morgeson and D. A. Hofmann. The structure and function of collective constructs: implications for multilevel research and theory development. Academy of Management Review 24(2): 249-265. (1999).

2. P.S. Churchland. Self-representation in nervous systems. Science 296: 308-310. (2002).

3. A. Damásio. The feeling of what happens: body and emotion in the making of consciousness. New York, Harcourt Brace. (1999).

4. A. Damásio. Looking for Spinoza: joy, sorrow, and the feeling brain. London, William Heinemann. (2003).

5. S. Greenfield. The Private Life of the Brain: emotions, consciousness, and the secret of the self. New York, John Wiley \& Sons, Inc. (2000).

6. K.E. Weick and K.H. Roberts. Collective mind in organizations: heedful interrelating on flight decks. Administrative Science Quarterly 38(3): 357-381. (1993).

7. I. Mitroff. Stakeholders of the Organizational Mind. San Francisco, Jossey-Bass. (1983). 
8. C.R. Schwenk. Strategic Decision Making. Journal of Management 21(3): 471493. (1995).

9. D.A. Levinthal and C. Rerup. Bridging Mindful and Less Mindful Perspectives on Organizational Learning. (2004).

10. P. Pawlowsky. Management science and organizational learning. Handbook of Organisational Learning and Knowledge. M. Dierkes, A. Berthoin-Antal, J. Child and I. Nonaka, Oxford University Press. (2001).

11. E.C. Hoogerwerf and A.-M. Poorthuis. The network multilogue: A Chaos approach to organizational design. Journal of Organizational Change Management 15(4): 382-390. (2002).

12. F.M.V. Ejjnatten and L. W. L. Simonse. Organizing for Creativity, Quality and Speed in Product Creation Processes. Quality And Reliability Engineering International 15: 411-416. (1999).

13. B. Sen. Organisational mind: Response to a paradigm shift in the Indian business environment. International Journal of Human Resources Development and Management. 3(1). (2003).

14. T. Ambler and C. Styles. Connecting Firm-level Learning with Performance, Center for Marketing, London Business School. (2002).

15. C.B. Gibson. From Knowledge Accumulation to Accommodation: cicles of collective cognition in work groups. Journal of Organizational Behavior 22: 121134. (2001).

16. E. Vaast and G. Walsham. Representations and actions: the transformation of work practices with IT use. Information and Organization 15: 65-89. (2005).

17. Hatch, M. J. and M. Schultz "The dynamics of organizational identity." Human relations 55(8): 989-1018. (2002).

18. R.J. Boland, R. V. Tenkasi, et al. Designing Information Technology to Support Distributed Cognition. Organization Science 5(3): 456-475. (1994).

19. D. Ravasi and J. v. Rekom. Key issues in organizational identity and identification theory. Corporate Reputation Review 6(2): 118-132. (2003).

20. V, Anand, C. C. Manz, et al. An organizational memory approach to information management. The academy of management review 23(4): 797-809. (1998).

21. H.R. Nemati, D. M. Steiger, et al. Knowledge warehouse: an architectural integration of knowledge management, decision support, artificial intelligence and data warehousing. Decision Support Systems 33: 143-161. (2002).

22. Y. Merali. The role of boundaries in knowledge processes. European Journal of Information Systems 11(1). (2002). 